\title{
A Digital Pressure Sensor in Soaring Vehicles and Gliders
}

Key Words: Vertical speed; pressure; digital sensors; gliders; Unmanned Aerial Vehicle (UAV); digital signal processing algorithms; Microcontroller units (MCUs).

Abstract. This paper describes a method for software optimization of digital sensor for vertical speed estimation applications. The system solution is based on Digital Signal Processing (DSP) algorithms discretized and adjusted for software implementation. Responsiveness, sensitivity and algorithm performance are considered. The presented method in this paper is tested in real flight conditions on an Unmanned Aerial Vehicle (UAV) and piloted paraglider. A two widely used digital pressure sensors, manufactured by two leading sensor producers, are used in the experiments.

\section{Introduction}

Numerous accounts of birds soaring without flapping their wings, ranging from observations by Leonardo da Vinci [1] to Octave Chanute [2] can be found in the literature. Birds circling in thermals are popular examples of advantageous use of atmospheric energy. Nowadays different types of gliders are soaring thermals and reaching hundreds of kilometres and dozens hours in the air in a single unpowered flight.

There are different forms of energy in the atmosphere boundary layer. Some forms have more strength than others, the latter being more predictable. Such dynamical forms of energy we observed on: i). Ridges (and orographic lift, where the slopes of hills and mountains deflect wind); ii). Atmospheric thermals (uneven heating of the ground, which produces buoyant instabilities); iii). Waves (long period oscillations of the atmosphere, which occur in the lee of large mountain range).

During soaring, the lift identification method where the updraft velocity is estimated is using the vehicle energy rate of change and the vehicle speed polar. The energy gain from the atmosphere is in the form of altitude gain caused by the updraft velocity. The vertical velocity component of the atmospheric thermal acting on the aircraft, when it is sufficient, will cause the aircraft to ascend [4].

The most widely used method for vertical speed indication is the measurement of the air pressure. Numerous companies now offer low cost piezo resistive sensors with digital output on the market. The use of these types of sensors can be for aerial vehicles applications due to the requirements for small package size, lightweight and low energy consumption, easy implementation and interfacing. However, accuracy requirements usually cannot be satisfied. In this context, this paper focuses on using piezoresistive pressure sensors with digital output that can mea

\section{A. Shamliev}

sure fast enough pressure, in order to provide real information about the vertical speed momentum.

The dependence, which evaluates the vertical velocity, is derived from the barometric eponent

(1) $p(z)=p_{0} e^{\frac{-z}{z_{0}}} \quad z_{o}=\frac{k T}{g m_{0}}$

Where $\mathrm{p}(\mathrm{z})$ is the pressure in altitude, $p_{0}$ is the sea level altitude air pressure, $\mathrm{T}$ is the temperature and the other elements in the equation are constants. The exponential element $e^{-z}$ is converted to the Taylor polynomial:

(2) $\frac{d z}{d t}=-\frac{z_{0}}{p_{0}} \frac{d v(p)}{d t}$.

A short literature search provides a wide set of solutions as digital signal processing is widely explored in many technical areas. However, detailed research of using digital pressure sensors for unpowered aerial vehicle altitude estimation is still an underdeveloped area. Those sensors usually are used as a support sensor data to more complementary Inertial Measurement Units (IMU) and Global Navigation Systems (GPS). The assumption of hydrostatic equilibrium is generally valid, provided the effects of a short-term period. In the real atmosphere significant variations are also observed in pressure, temperature and even lapse rate. Absolute altitude information cannot be easily obtained; in particular, it is necessary to know, inter alia, the local sea level pressure, which may differ from standard pressure. Equation (2) still applies in cases where the pressure and the temperature differ from those of the standard: the relative change in pressure and the actual temperature determine the change in altitude regardless of the altitude. The change in altitude also is be important in vertical speed indication for soaring flights [3].

The purpose of this work is to demonstrate the capabilities of piezo resistive pressure sensors with digital output and to propose a software-implemented optimization method that provides suitable results for UAVs.

\section{Digital Pressure Measurements}

The Integrated Pressure Sensors (IPS) have trimmed outputs, built-in temperature compensation and an amplified single-ended output, which make them compatible with Analog to Digital converters (A/D's) on low cost micro-controllers. Although 8-bit A/D's are most common, higher resolution, A/D's are becoming increasingly available. With this higher resolution A/D's, the noise that is inherent in the piezo-resistive bridges becomes a design consideration [4]. 
Digital filters are implemented as a computer program that transforms sampled data. In other words, a filter that we would like to use should be discretised.

Measuring the parameters of dynamically changing atmosphere for the purpose of the convection events evaluation is related to fast measurements and calculations. In this work, the developed measurements system consists of the following hardware components:

i). Launchpad TI MSP430FR5994 and SD Card development kit developed by Texas Instruments [5]; ii). Digital sensor BME280 produced by Bosh Sensortec [6] and MS5611 produced by TE Connectivity [7];

iii). Standard Li-ion battery suppling the system with 3.3 V through microchip MCP series LDO (low drop out regulator) [8].

The following software is implemented in the measurement system:

i) Texas instrument library for SD Card recording [5];

ii) Bosh Sensortec library for interfacing the BME280 sensor [6].

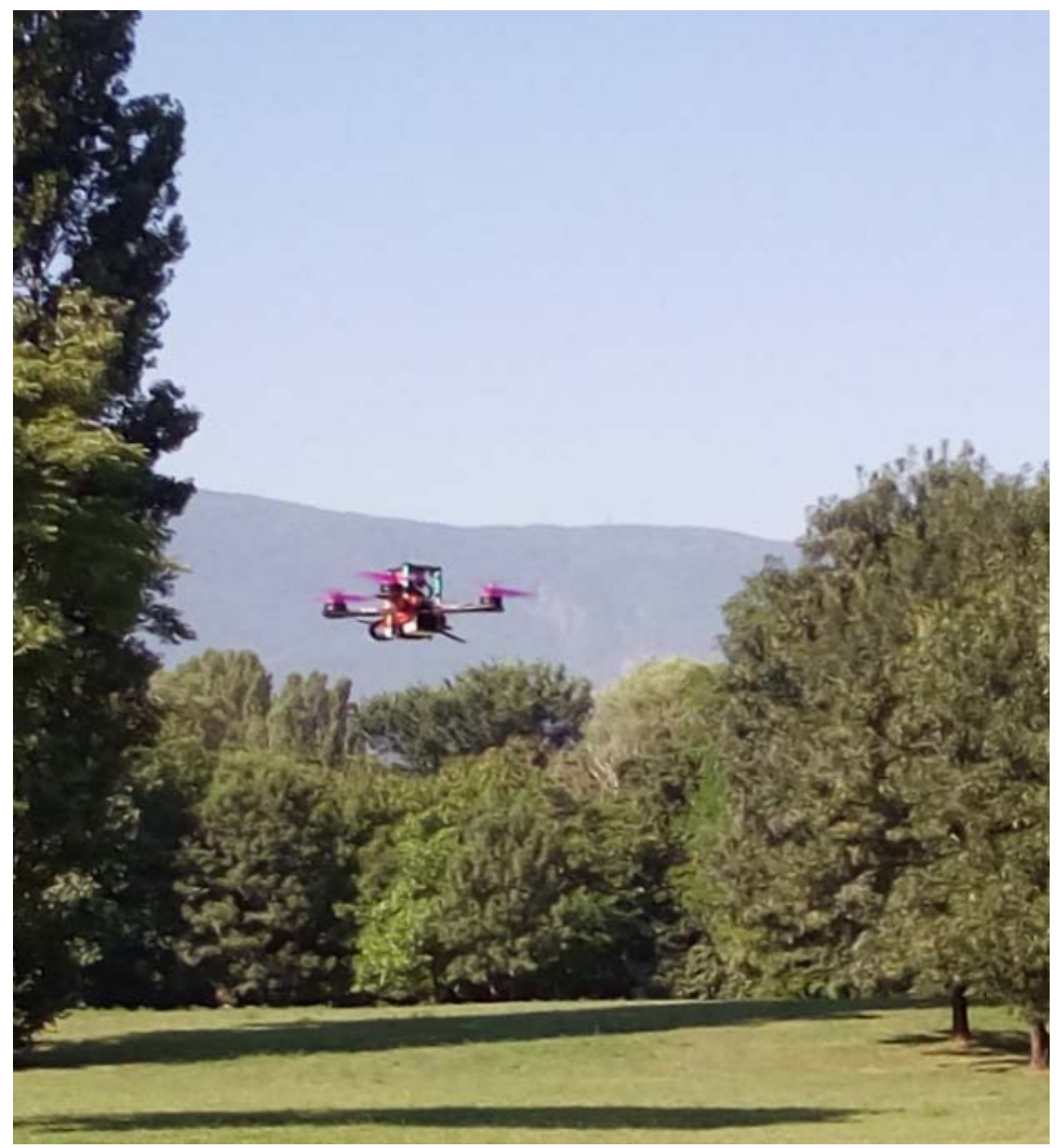

Figure 1. Quadcopter drone loaded with a measurement system

Several measurement seasons are performed on radio controlled quadcopter drone platform and piloted paraglider. The quadcopter drone performed 150 meters vertical ascending and strong thermal conditions in addition experimental measurements are performed in indoor condition in a laboratory.

A sample unpowered flight altitude (pressure) profile is shown in figure 2. The data is recorded by the developed measurement system. It is obvious that lower the pressure is, higher the altitude is. Because the measurements are made airborne at unpowered vehicles, the drop of the pressure is because of vertical altitude gain acquiring from convectional process in the atmosphere (thermal convection or just thermals).
In General, the two dominant types of noise in a piezoresistive integrated pressure sensor are (white) noise and 1/f (flicker noise) [9].

Based on the performed measurements, the effect of the white nose is amplified in outdoor and flight conditions compared with the laboratory experiments, because additional noises are added as atmosphere disturbance, mounting issues and heat sources.

Since random (or 'white') noise is distributed over all frequencies, and signal (measured pressure variation) is limited to low frequencies, a reduction of high frequency components will improve the signal/noise ratio [7]. Most of the producers of all types of digital sensors (not only limited to those used in this work nor pressure ones), recom- 


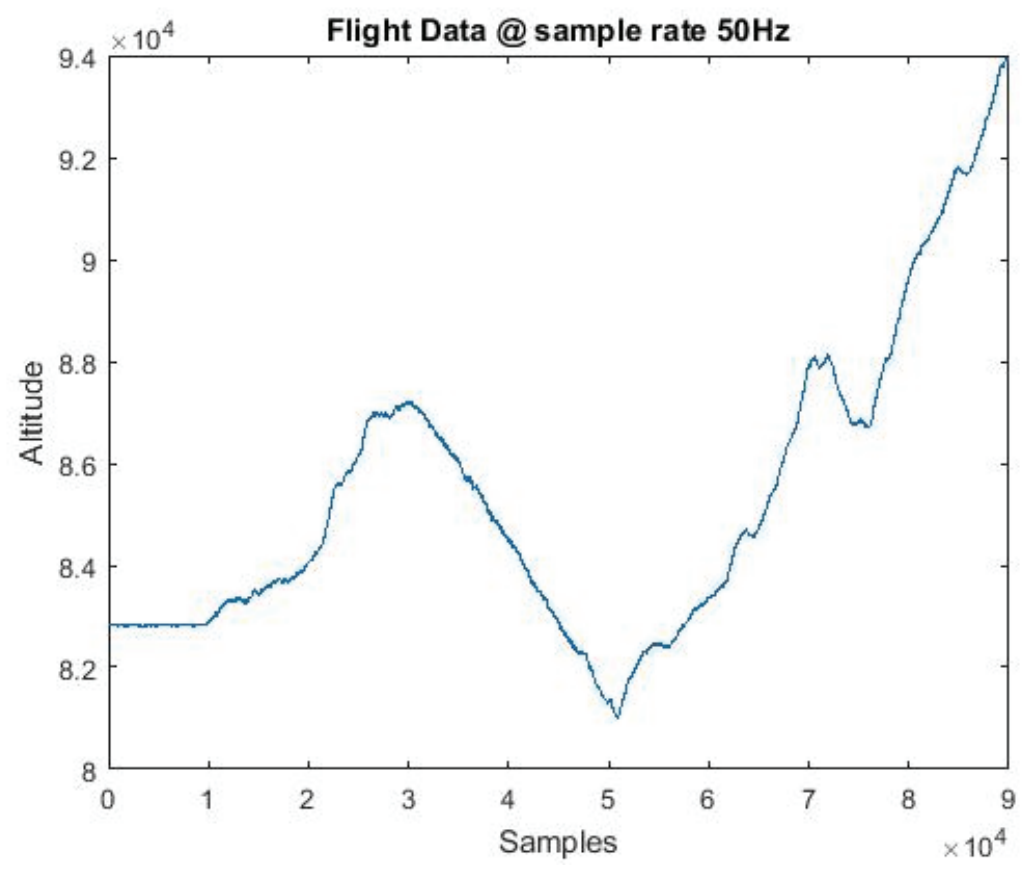

Figure 2. Unpowered flight altitude (pressure) profile

mend to use simple discreet first order infinite impulse filter (IIR) $[8,9]$. In addition to this, experiments and explorations are performed in order to improve the results from the sensors.

As highest odder discrete filter is used, the results are clear and accurate, but a higher delay of the signal is generated. On the other hand, smoothing algorithms as regressive functions and extrapolations require more samples, leading to losing of sampling rate or higher response time of the results.

Three criteria, by which the quality of the results is evaluated, are reviewed below:

- Time response.

- Computational resources.

- Accuracy.

Unfortunately, all these criteria are connected, and it is not possible to improve any of them without affecting the other two (figure 3).

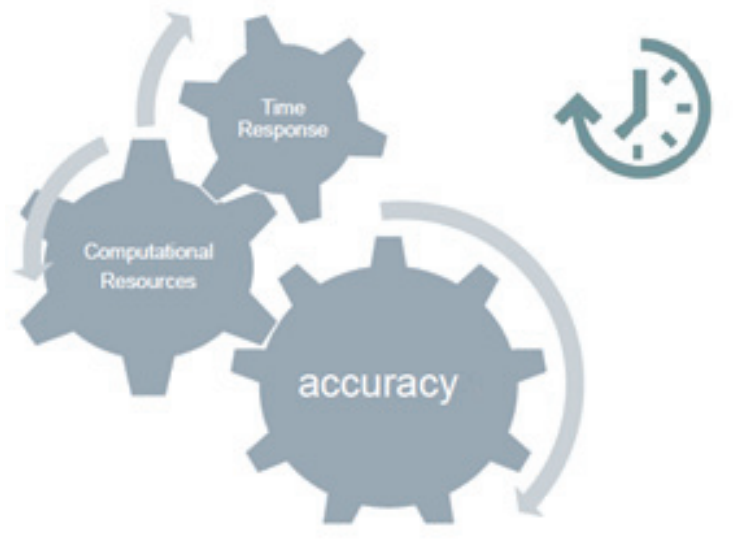

Figure 3. Time vs accuracy vs computational resources
The main task of this work can also be defined as finding the configuration of the above-mentioned criteria in order to be best optimized and adjusted for soaring flights applications.

It is well-known that digital pressure sensors contain additional low frequency noise. The concentration of noise energy in the low frequency region of the spectrum can be explained as the consequence of, e.g. thermal gradients and air flow in the sensor vicinity [10]. In measuring the absolute altitude, these low frequency pressure fluctuations are correctly assigned as noise. However, for the purpose of measuring vertical gain of velocity, this information not only should not be defined as a noise, but must be considered as important information.

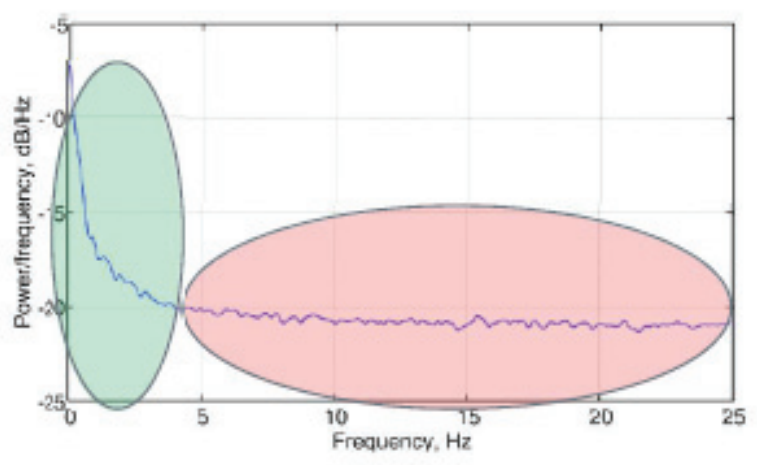

Figure 4. Pressure altitude noise spectrum [10]

Most of the thermals we encounter are 30 meters or less in diameter. Some thermals especially those in weaker conditions - can be quite larger of course. However, when we find lifting areas larger than 100 meters we should suspect a source of lift other than pure thermals. [11] On the 
other hand, fluctuations less than $10 \mathrm{~m}$ (turbulent air) could be considered as unusable (or technically ineffective).

As an average cruising speed of the tested vehicles $10 \frac{\mathrm{m}}{\mathrm{s}}$ it could be assumed that a response time ("lag") of the system is optimal. Further, this response time (that at first time cold be considered as disadvantage) could be used for filtering the small fluctuations.

\section{Optimisation Software Methods}

Based on the stated in the introduction paragraphs, in this work a separate infinite impulse response filter (IIR) followed by $4^{\text {th }}$ order Butterworth filter are implemented, followed by Kalman filtering. That cascade configuration of the Kalman filter allows us to benefit from higher order recursive filters, while keeping the response time of the system.

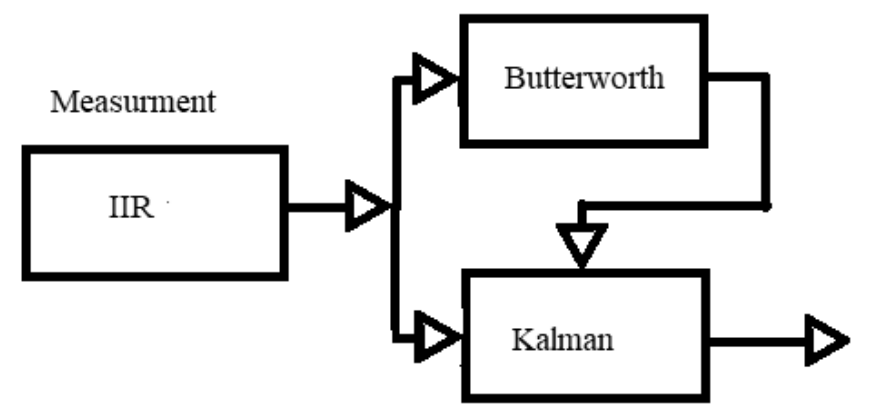

Figure 5. Block scheme of the optimization-filtering model

The discrete IIR Filter

$$
H(z)=\frac{\sum_{i=0}^{p} b_{i} z^{-i}}{1+\sum_{j=1}^{Q} a_{j} z^{-i}}
$$

where $b_{i}$ are the feedforward filter coefficients, $Q$ is the feedback filter order, $a_{j}$ are the feedback filter coefficients. In addition, because a first order non-recursive filter is used:

(4)

$$
H(z)=\frac{z_{t-1} b_{t-1}+z_{t}}{b_{t}}
$$

where $t$ is the sample and $\mathrm{b}$ is the filter coefficient.

The 'Kalman Filter' addresses the general problem of trying to estimate the state $H_{x}, H_{y}$ of a discrete-time controlled process that is governed by the linear stochastic difference equation

(5) $x_{k}=A x_{x-1}+B u_{k}+w_{k-1}$

with a measurement $z \in R^{m}$ that is

(6) $z_{k}=H x_{k}+v_{k}$.

The variables $w_{k}$ and $v_{k}$ represent the process and measurement of the noise, respectively. They are assumed to be independent of each other, white, and with normal probability distributions

$$
\begin{aligned}
& \text { (7) } \mathrm{p}(\mathrm{w}) \sim \mathrm{N}(0, \quad \mathrm{Q}) \\
& \text { (8) } \mathrm{p}(\mathrm{v}) \sim \mathrm{N}(0, \quad \mathrm{R}) \text {. }
\end{aligned}
$$

$\mathrm{Q}$ is the process noise covariance and $\mathrm{R}$ is the measurement noise covariance. Equation (5) is similar to the standard state differential equation

(9) $x_{k}=A_{x}+B_{u}$

where $x_{k}$ is the state vector and $\mathrm{u}$ is the input or driving function, the only difference being that equation (5) is a system whose state vector is sampled for discrete time state, whereas equation (5) is sampled for continuous time state.

The $n \times n$ matrix $A$ in the difference equation (5), relates the state at the previous time step $\mathrm{k}-1$ to the state at the current time step $\mathrm{k}$, in the absence of a driving function or a process noise. The $\mathrm{n} \times 1$ matrix $\mathrm{B}$ relates the optional control input $u \in R^{n}$ to the state $\mathrm{x}$. The $\mathrm{m} \times \mathrm{n}$ matrix $H$ in equation (6) relates the state $x_{k}$ to the measurement $\mathbf{z}_{k}$.

An initial estimate of the process at some point $t_{k}$ is assumed, and this estimate is based on our knowledge of the process prior to $t_{k}$. Let this a priori estimate be denoted by $\hat{x}_{k}{ }^{-}$, where the "hat" denotes estimate, and the "super minus" reminds us that this is the best estimate we have prior to assimilating the measurement at $t_{k}$. Assuming that the error covariance matrix associated with $\hat{x}_{k}{ }^{-}$is also known, and then the estimation error is defined as

(10) $\hat{\mathrm{e}}_{\mathrm{k}}^{-}=x_{x}-\hat{x}_{k}^{-}$.

The associated covariance matrix is:

$$
P_{k}^{-}=E\left[e_{k}^{-} e_{k}^{-T}\right]=E\left[\left(x_{x}-\hat{x}^{-}\right)\left(x_{k}-\hat{x}^{-}\right)^{-T}\right]
$$

Since we have assumed a prior estimate $\hat{x}^{-}$, we use $z_{k}$ to improve the prior estimate, by the following equation:

$$
\hat{x}_{k}=\hat{x}_{k}^{-}+K_{k}\left(z_{k}-H_{k} \hat{x}_{k}^{-}\right)
$$

where $\hat{x}_{k}$ is the updates estimate and $K_{k}$ is the blending factor or 'Kalman' gain that minimizes the a posteriori error covariance equation (9).

$$
P_{k}=E\left[e_{k} e_{k}^{T}\right]=E\left[\left(x_{k}-\hat{x}\right)\left(x_{k}-\hat{x}\right)^{T}\right]=\left(I-K_{k} H_{k}\right) P_{k}^{-}
$$

The 'Kalman' gain, which minimizes the mean-square estimation error, is given by

(14) $K_{k}=P_{k}^{-} H_{k}^{T}\left(H_{K} P_{k}^{T}+R_{k}\right)^{-1}$.

Then the next step measurement $\hat{x}_{k+1}^{-}$is estimated, the error covariance $\hat{P}_{k+1}^{-}$calculated and the process is repeated (figure 6)

$$
\begin{gathered}
\hat{x}_{k+1}^{-}=A_{k} \hat{x}_{k}+B_{k} u_{k} ; \\
\hat{P}_{k+1}^{-}=A_{k} P_{k} A_{k}^{T}+Q_{k} .
\end{gathered}
$$

To simplify the explanations, by measurements or sensor data means the data acquired after the IIR filter and the data acquired after the Butterworth filter is simplified to 


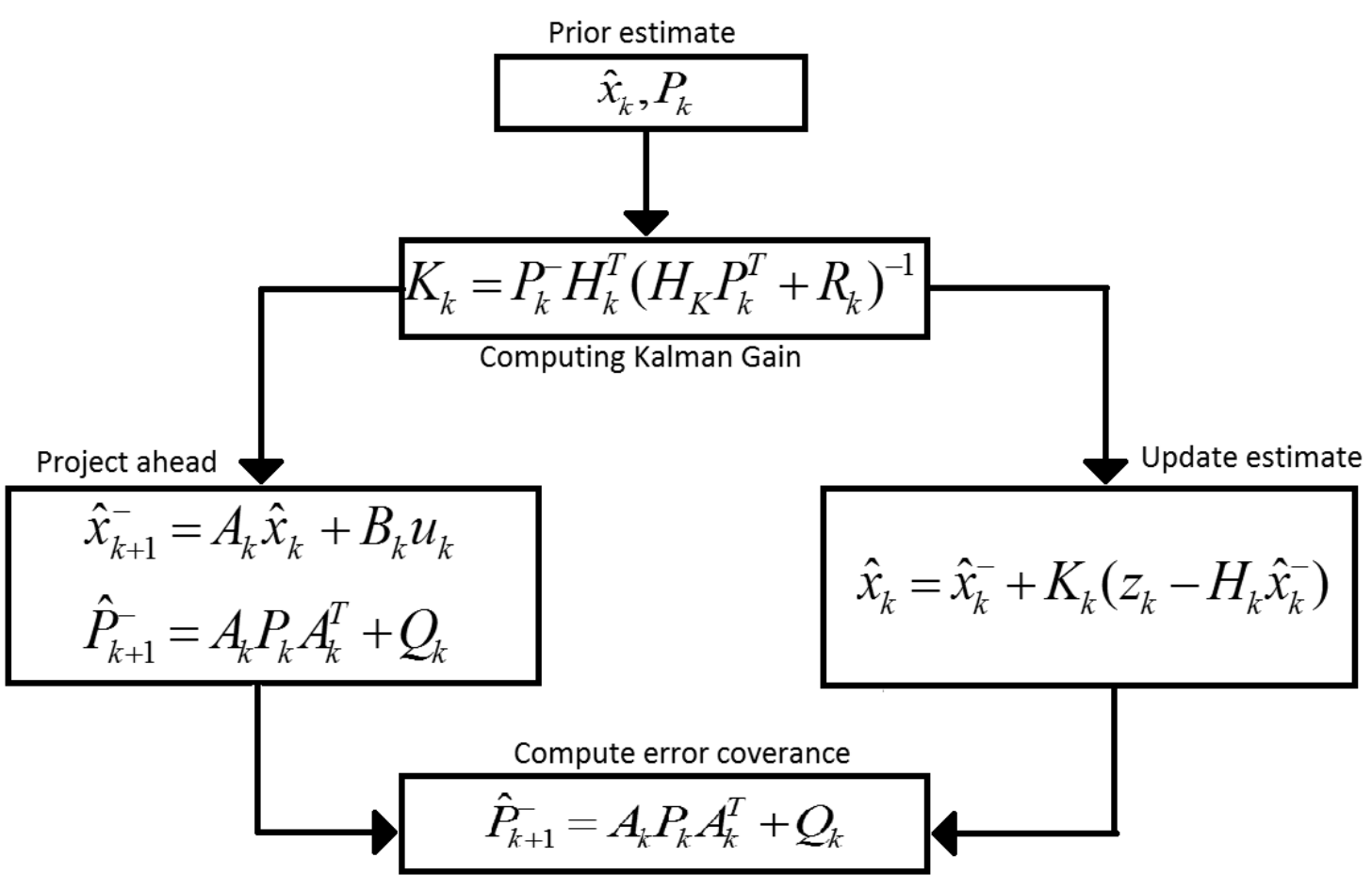

Figure 6. The Kalman Loop

filtered data in the next paragraphs.

These are two main steps - state update and "Kalman" update. The state update is used every $d t$ (every sample)with a pressure measurement. It updates the current altitude and rate estimate.

The "Kalman" update is after the Low pass filtration. To simplicity the in the next paragraph sensor measurement is equal to the result and filter data means the result of the Butterworth filter.

The covariance matrix $P$ is updated at every time step $(d t)$ and it is determined how well Low pass filter are tracking the actual state. The initial state of covariance matrix is

$$
P=\left[\begin{array}{ll}
1 & 0 \\
0 & 1
\end{array}\right] \text {. }
$$

The measurement covariance noise is represented by matrix $\mathrm{R}$. The process covariance noise is represented by $2 \times 2$ matrix $Q$. In this case, it indicates how much we trust the sensor measurement relative to the result after the filter is applied

(18) $\mathrm{Q}=\left[\begin{array}{cc}Q_{s} & 0 \\ 0 & Q_{l p}\end{array}\right]$.

$Q_{\text {sensor }}-$ covariance of the measurement/sensor;

$Q_{\text {filter }}-$ covariance of the filter.

The state vector is and the estimation state vectors are $\mathrm{X}=$ [ sensor, $]$ and $\dot{\mathrm{X}}=$ [ sensor, filter $]$.

The covariance matrix $\mathrm{P}$ is updated by the next expressions. In the beginning, it is computed the derivative of the covariance matrix

(19) $\dot{\mathrm{P}}=\mathrm{A}^{*} \mathrm{P}+\mathrm{P}^{*} \mathrm{~A}^{\prime}+\mathrm{Q}$.
The Jacobian matrix A calculated of $\dot{\mathrm{X}}$ with respect to the state vector $\mathrm{X}$ :

(20) $\quad \mathrm{A}=\left[\begin{array}{ll}\frac{\mathrm{d}(\text { sensor })}{\mathrm{d}(\text { sensor })} & \frac{\mathrm{d}(\text { sensor })}{\mathrm{d}(\text { filter })} \\ \frac{\mathrm{d}(\text { filter })}{\mathrm{d}(\text { sensor })} & \frac{\mathrm{d}(\text { filter })}{\mathrm{d}(\text { filter })}\end{array}\right]$

Then it is computed the expansion of $A=\left[\begin{array}{rr}0 & -1 \\ 0 & 0\end{array}\right]$ multiplied by $\mathrm{P}$ and $\mathrm{P}$ multiplied by $\mathrm{A}^{\prime}=\left[\begin{array}{rr}0 & 0 \\ -1 & 0\end{array}\right]$. This is then added to the diagonal elements of the process covariance noise matrix $\mathrm{Q}$, which are $Q_{\text {sensor }}$ and $Q_{\text {filter }}$

$$
\begin{aligned}
& \dot{\mathrm{P}}_{0}=Q_{\text {sensor }}-\mathrm{P}_{01}-\mathrm{P}_{10} \text { and } \\
& \dot{\mathrm{P}}_{1}=-\mathrm{P}_{11} ; \dot{\mathrm{P}}_{2}=\mathrm{P}_{11} ; \dot{\mathrm{P}}_{3}=-\mathrm{Q}_{l p} .
\end{aligned}
$$

The updated estimate and covariance matrix $\mathrm{P}$

$$
\mathrm{P}=\left[\begin{array}{ll}
\dot{\mathrm{P}}_{0} * d t & \dot{\mathrm{P}}_{1} * d t \\
\dot{\mathrm{P}}_{2} * d t & \dot{\mathrm{P}}_{3} * d t
\end{array}\right]
$$

The $\mathrm{C}$ matrix is a [1×2] (measurements $\mathrm{x}$ states) matrix that is the Jacobian matrix of the measurement value sensor ${ }_{m}$ with respect to the states vector $\mathrm{X}$ [sensor, filter]. In this case, $\mathrm{C}$ is

$$
\text { (23) } \mathrm{C}=\left[\frac{\mathrm{d}\left(\text { sensor }_{m}\right)}{\mathrm{d}(\text { filter }))}, \frac{\mathrm{d}\left(\text { sensor }_{m}\right)}{\mathrm{d}(\text { filter })}\right]=\left[\begin{array}{ll}
1 & 0
\end{array}\right] \text {. }
$$




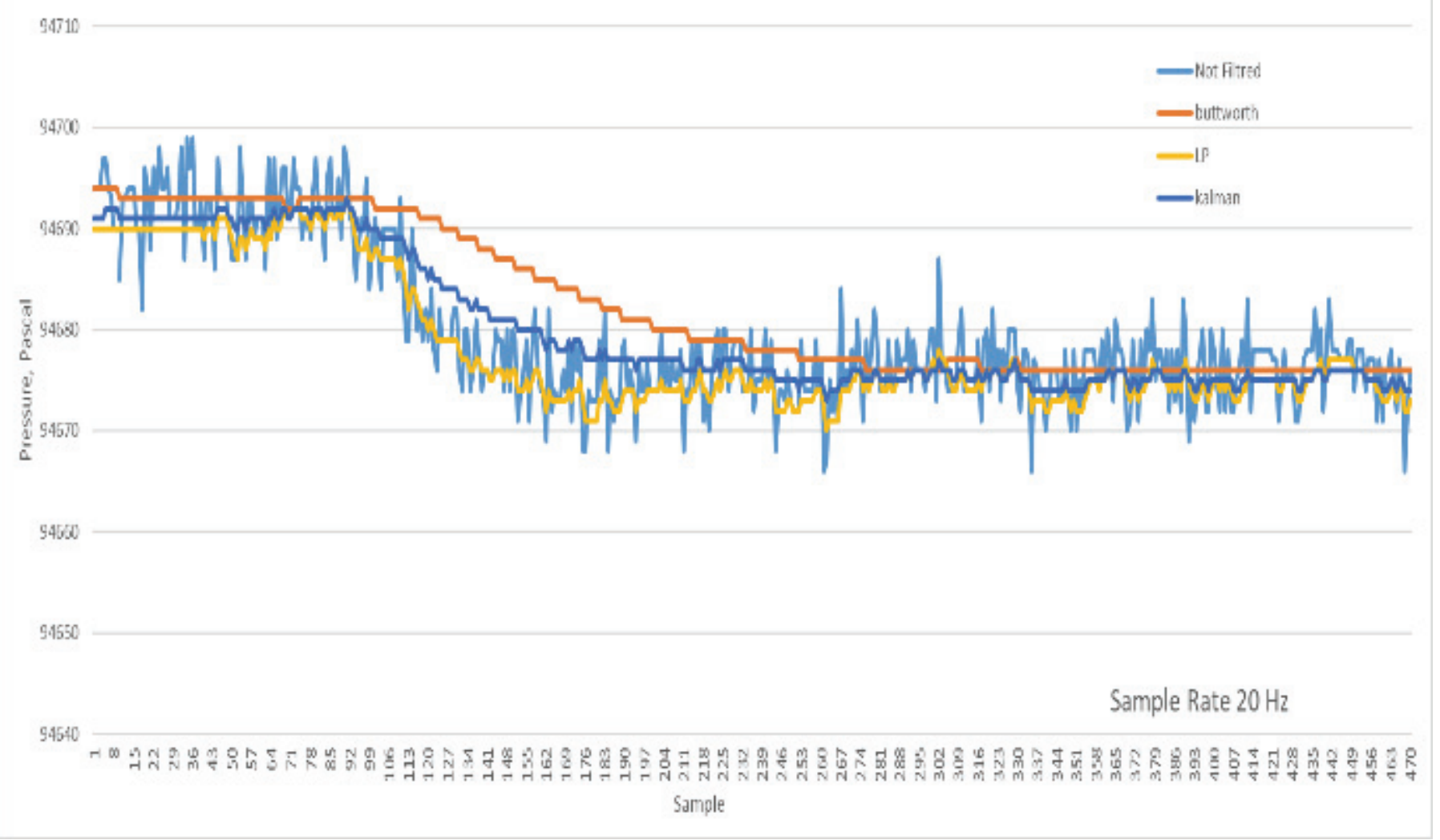

Figure 7. Optimization result

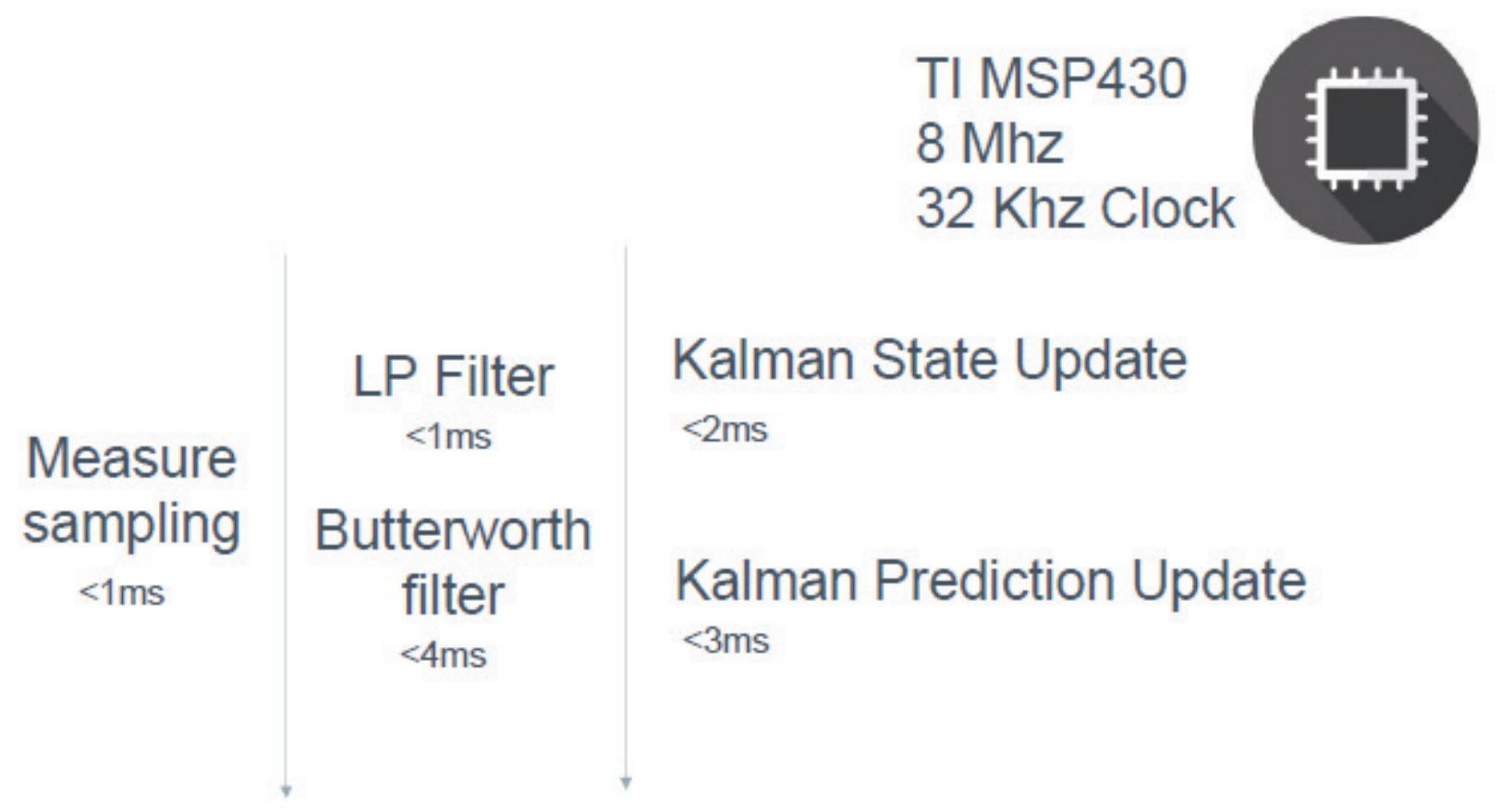

Figure 8. Computational resources 
The first element of the Jacobian matrix of the measurement value sensor ${ }_{m}-\mathrm{C}_{0}=\left[\frac{\mathrm{d}\left(\text { sensor }_{m}\right)}{\mathrm{d}(\text { filter }))}\right]=1$, shows how the state measurement directly relates to the state estimate.

The next step is computed the Kalman filter gains.

(24) $\mathrm{K}=\mathrm{P} \mathrm{C}^{\prime} \operatorname{inv}(\mathrm{E})$;

(25) $\mathrm{K}_{[2 \times 1]}=\mathrm{P}_{[2 \times 2]} \mathrm{C}_{[2 \times 1]}^{\prime} \operatorname{inv}(\mathrm{E})_{[1 \times 1]}$

For Kalman filter gains we have

$$
\begin{gathered}
\mathrm{K}_{0}=\frac{\mathrm{PC}_{t_{0}}}{\mathrm{E}} ; \\
\mathrm{K}_{1}=\frac{\mathrm{PC}_{t_{1}}}{\mathrm{E}} .
\end{gathered}
$$

The updated covariance matrix:

$$
\begin{aligned}
& \mathrm{P}=\mathrm{P}-\mathrm{K} \text { C P } \\
& \mathrm{P}_{[2 \times 2]}=\mathrm{P}_{[2 \times 2]}-\mathrm{K}_{[2 \times 1]} \mathrm{C}_{[1 \times 2]} \mathrm{P}_{[2 \times 2]} .
\end{aligned}
$$

It is computed $t_{[1 \times 2]}=\mathrm{C} \mathrm{P}$. Note that:

$$
\begin{aligned}
& \mathrm{t}_{0}=\mathrm{C}_{00} * \mathrm{P}_{00}=\mathrm{PC}_{\mathrm{t} 0} \\
& \mathrm{t}_{1}=\mathrm{C}_{0} * \mathrm{P}_{01} ; \\
& \mathrm{P}_{00}=\mathrm{P}_{00}-\mathrm{K}_{0} * \mathrm{t}_{0} \\
& \mathrm{P}_{01}=\mathrm{P}_{01}-\mathrm{K}_{0} * \mathrm{t}_{1} \\
& \mathrm{P}_{10}=\mathrm{P}_{10}-\mathrm{K}_{1} * \mathrm{t}_{0} \\
& \mathrm{P}_{11}=\mathrm{P}_{11}-\mathrm{K}_{1} * \mathrm{t}_{1}
\end{aligned}
$$

Finally, it is updated state estimate:

$$
\begin{aligned}
& \mathrm{X}=\mathrm{X}+\mathrm{K} * \text { Err; } \\
& \mathrm{X}_{[1 \times 2]}=\mathrm{X}_{[1 \times 2]}+\mathrm{K}_{[2 \times 1]} * \operatorname{Err}_{[1 \times 1]^{*}}
\end{aligned}
$$

The "Err" is a measurement of the difference in the measured state and the estimate state.

$$
\begin{aligned}
& \text { sensor }_{\text {err }}=\text { sensor }_{\text {measure }}-\text { pressure; } \\
& \text { Pressure }=\text { pressure }+\mathrm{K}_{0} * \text { sensor }_{\text {err }} .
\end{aligned}
$$

\section{Results}

The results are shown on figure 7. In general, the proposed method combines fast response capability of IIR and the accuracy of the higher order Butterworth filter.

The result shown are after applying of the following coefficients: IIR Filter $-a_{j}=4$ and Kalman covariance coefficients $q_{\text {sensor }}=q_{\text {filter }}=0.003$. The coefficients selection is based on the fact stated in the introduction, to keep no more than one second time delay of the system. If the sensor measurement rate is higher, IIR coefficient could be bigger.

Using the Texas Instruments Code Composer Studio debugging tools, the computational times of the proposed method are shown on figure 8 .

\section{Conclusion}

Multiple digital signal processing (DSP) algorithms can be applied in this specific application. Instead of Butterworth filter, other recursive filtration is acceptable. In addition, other data fusion method of complementary filter can replace the Kalman filter. Finally, with the maturation and advancement of semiconductor manufacturing technology, the digital pressure sensors are increasing their characteristics - sample rates, accuracy, and functionality as integrated filtering modes. Thus it can be power and resource efficient to unload the microcontroller unit (MCU) processing and processing the data.

However, based on up to 25 hours of measurements during flights on paraglider and micro quadcopter UAV, the described method is considered as optimal, taking into account: i) Easy discretisation and software implementation; ii) Software algorithm that is capable for executing using small resources of an average MCU that are on the market nowadays.

This paper proves the use of the low cost piezo resistive pressure sensors with digital output as a reliable and robust way for vertical speed estimation. The presented method for a software optimization proposes further measurement accuracy.

The scope of this work is part of research on evaluation of the boundary layer of the atmosphere based on a multisensory system for unpowered aerial vehicles application.

\section{Acknowledgements}

I am grateful to eng. Blagovest Hristov, Institute of Robotics, Bulgarian Academy of Sciences for performing several test flights on quadcopter UAV, constructed by him.

I also thank my supervisor Assoc. Prof. Maya Dimitrova for her help in the preparation of this paper.

\section{References}

1. Jackman, W. J. and T. H. Russell. Flying Machines: Construction and Operation. The Charles C. Thompson Co., Chicago, USA, 1910, Chapter XX.

2. Laurenza, D. Leonardo on Flight. Giunti Press, Milan, Italy.

3. Husak, T., J. Jakovenko. Pressure Sensor Data Processing for Vertical Velocity Measurements. NSTI Nanotech 2008, Department of Microelectronics, Czech Technical University Prague, 02-03.

4. Cone, C. D. The Design of Sailplanes for Optimum Thermal Soaring Performance. Technical Report TND 2052, NASA, 1964.

5. https://ae-bst.resource.bosch.com/media/_tech/media/datasheets/ BST-BMP280-DS001-18.pdf.

6. http://www.ti.com/lit/ug/slau678a/slau678a.pdf .

7.http://www.te.com/commerce/DocumentDelivery/ DDEController? Action $=$ srchrtrv\&DocNm=MS 5611 01BA03\&DocType=Data + Sheet\&DocLang=English . 
8. http://ww1.microchip.com/downloads/en/DeviceDoc/22008E.pdf. 9. Schultz, W and A. Reodique. Noise Considerations for Integrated Pressure Sensors. NXP Semiconductors, 01.

10. Angelo Maria Sabatini, Vincenzo Genovese. A Stochastic

\section{Manuscript received on 08.01.2018}

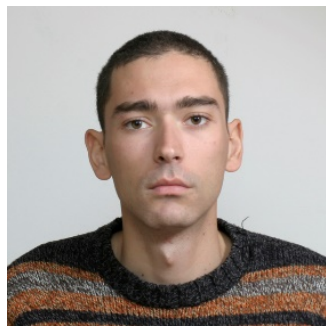

Alexander Shamliev received his M.S. degree in Aviatiion Technologies in the Tehnical University Sofia, in 2012. He is currently PhD student at the Institute of Robotics of BAS (IR-BAS).

Contact: Institute of Systems Engineering and Robotics

Bulgarian Academy of Sciences Acad. G. Bonchev St., bl. 2,

P.O. Box 79

1112 Sofia, Bulgaria e-mail: a.shamliev@gmail.com
Approach to Noise Modeling for Barometric Altimeters, Sensors ISSN 1424-8220.

11. Dennis, Pagen. Understanding the Sky. 